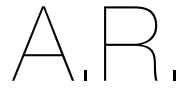

ARTIGO DE REVISÃO

${ }^{1}$ Escola Superior Agrária

de Coimbra, Instituto Politécnico de Coimbra, 3045-601 Coimbra,

Portugal

2 Unidade de I\&D CERNAS, Escola Superior Agrária de Coimbra, Instituto Politécnico de Coimbra,

3045-601 Coimbra Portugal

${ }^{3}$ Clínica Médica e Dentária Conímbriga XXI, 3150-230 Condeixa-aNova, Portuga

"Endereço para correspondência

Goreti Botelho Escola Superior Agrária de Coimbra, Instituto Politécnico de Coimbra, 3045-601 Coimbra, Portuga goreti@esac.pt

Histórico do artigo:

Recebido a 2 de junho de 2021 Aceite a 30 de setembro de 202

\title{
ALIMENTAÇÃO À BASE DE PLANTAS: UMA REVISÃO NARRATIVA
}

\author{
PLANT-BASED DIETS: A NARRATIVE REVIEW
}

Miguel Lima'; Rui Costa ${ }^{1,2}$; Jorge Lameiras ${ }^{3}$; Goreti Botelho ${ }^{1,2}$

\section{RESUMO}

A alimentação é uma necessidade básica para todos os indivíduos importante para a sua saúde e bem-estar. A adoção de dietas tendencialmente vegetarianas, encontra justificação não só em argumentos de saúde e bem-estar, nomeadamente tendo em consideração alguns efeitos prejudiciais de dietas omnívoras na saúde, mas também em argumentos relacionados com o ambiente, e de ideologia de vida. Os principais objetivos deste trabalho de revisão narrativa centram-se em três vertentes interligadas e de elevada atualidade: (1) rever os argumentos acerca do papel das dietas à base de plantas na prevenção de doenças crónicas e no bem-estar humano; (2) enquadrar as dietas à base de plantas enquanto dietas com reconhecido envolvimento na sustentabilidade social, económica e ambiental; e (3) destacar os principais produtos alimentares alternativos aos alimentos de origem animal e os desafios que a indústria alimentar enfrenta. Da literatura consultada, foram analisados 68 artigos científicos, permitindo concluir que a adoção de dietas com base vegetal tem evidenciado um papel relevante na prevenção de doenças crónicas, no bem-estar e no ambiente.

\section{PALAVRAS-CHAVE}

Bem-estar, Dieta baseada em plantas, Prevenção de doenças crónicas, Saúde, Sustentabilidade

\section{ABSTRACT}

Feeding is a basic need for all individuals relevant for their health and well-being. The adoption of vegetarian diets is justified not only in health and well-being arguments, namely considering some harmful effects of omnivorous diets on health, but also in arguments related to the environment and life ideology. The main objectives of this narrative review work focus on three interconnected and very actual topics: (1) to focus on the potential of plant-based diets in the prevention of chronic diseases and human well-being; (2) to frame plant-based diets as diets with recognized involvement in social, economic and environmental sustainability; and (3) to highlight the main alternative food products to foods of animal origin and the challenges facing the food industry. From the consulted literature, 68 scientific articles were analyzed, allowing to conclude that adopting a plant-based diet has played an important role in the prevention of chronic disease, well-being, and environmental sustainability.

KEYWORDS

Well-being, Plant-based diet, Chronic diseases prevention, Health, Sustainability

\section{INTRODUÇÃO}

Tem-se verificado um aumento do interesse do consumidor pelos padrões alimentares com base em alimentos de origem vegetal, invocando argumentos de saúde e bem-estar, ambientais e pela maior preocupação com os direitos e bemestar dos animais (1). As estimativas reportam um número de vegetarianos em tendência crescente a cada ano (2-4). Estes padrões assumem múltiplas formas, correspondendo a níveis diversos de exclusão de alimentos que não sejam de origem vegetal. A redução do consumo de carne tem sido ainda mais intensificada pelo crescente número de flexitarianos (uma dieta essencialmente vegetariana mas com inclusão ocasional de carne ou peixe), estimados em 7,4\% da população em 2019 (5).

O crescimento da população que adota alguma forma de alimentação com exclusão ou redução de produtos de origem animal, torna necessária a preparação ou especialização dos diferentes operadores que atuam neste âmbito, tanto ao nível da produção, como da comercialização e fornecimento de produtos de origem vegetal, bem como o reforço do conhecimento de nutricionistas e outros profissionais de saúde. É evidente a criação de incentivos à satisfação da procura destes modelos de alimentação, mediante a obrigatoriedade de uma "opção vegetariana" nos refeitórios escolares nacionais (art. ${ }^{\circ} 3^{\circ}$ da Lei n. ${ }^{\circ}$ 11/2017, de 17 de abrill (6). Para além das medidas já apresentadas, a DireçãoGeral da Saúde (DGS), no âmbito do Programa Nacional para a Promoção da Alimentação Saudável (PNPAS), elaborou três manuais sobre vegetarianismo. Importa ainda destacar que o ano de 2021 é o ano das frutas e legumes, a nível 
internacional, designado pela Assembleia Geral das Nações Unidas. É de realçar o investimento de cerca de 1 milhão de euros no projeto europeu EQVEGAN, que é uma aliança de competências setoriais composta por 15 instituições de 11 países, que pretende responder aos desafios colocados à indústria alimentar face ao crescimento do interesse do consumidor em produtos vegetais, disponibilizando formação inovadora e especializada, possibilitando assim um mercado mais qualificado e pronto a responder às exigências do consumidor (7).

Cada vez mais a saúde é assumida como parte integrante de um quadro holístico que engloba também a organização da sociedade e da economia e a salvaguarda do ambiente. A adoção de uma alimentação sustentável é fundamental para atingir os objetivos ambientais e para refletir sobre aspetos relacionados com a saúde pública e os custos ambientais da produção alimentar $(8,9)$.

Pretende-se neste trabalho: (1) rever os argumentos acerca do papel das dietas à base de plantas na prevenção de doenças crónicas e no bemestar humano; (2) enquadrar o uso das dietas à base de plantas num pensamento global de mudança para um paradigma de sustentabilidade ambiental; e (3) identificar produtos alimentares alternativos aos alimentos de origem animal, em particular à carne.

\section{METODOLOGIA}

Do ponto de vista metodológico procedeu-se à pesquisa nas bases de dados b-on, PubMed $^{\circledR}$ e ScienceDirect sem limitação de data ou país de realização dos estudos, mediante a utilização das seguintes palavraschave: "plant-based diet", "vegetarian diet" e "vegan diet". Após a leitura dos títulos de todos os artigos obtidos na pesquisa, identificaram-se 139 artigos potencialmente relevantes e após leitura parcial (resumo) ou integral, 68 foram selecionados na presente revisão narrativa, considerando a sua atualidade e adequabilidade do conteúdo. Adicionalmente, foram considerados livros de editor internacional e pesquisa de websites de alguns organismos nacionais e internacionais para pesquisa auxiliar, como a Estratégia Integrada para a Promoção da Alimentação Saudável (EIPAS) e a Organização Mundial de Saúde.

\section{Definição e Implicações de uma Alimentação de Base Vegetal}

O conceito de dieta baseada em plantas (DBP, do inglês Plant-Based Diet) não tem uma definição consensual (10). Alguns estudos sugerem que o consumo de produtos de origem animal deve ser reduzido mas não excluído (11, 12), o que é evidente nas opções de uso de alimentos como os ovos e os laticínios, ou até mesmo de pescado (alimentação pescetariana). Por outro lado, existe quem defenda que a DBP consiste na ingestão de frutas, produtos hortícolas, cereais integrais, frutos de casca rija, sementes, ervas e especiarias com total exclusão de produtos de origem animal, incluindo laticínios $(10,12)$. Nas DBP as escolhas alimentares devem, sobretudo, recair sobre alimentos com baixo processamento, e priorizando alimentos integrais, exigência que não é comum a todas as dietas, nomeadamente vegetarianas não veganas.

O conceito de DBP é amplo na variedade de formas que abrange, e centra-se na composição da dieta e não em aspetos morais ou identitários de grupo, frequentemente associados às dietas vegetarianas e veganas (13), o que pode ser importante para a discussão objetiva acerca do seu interesse nutricional.

A terminologia "dieta vegetariana" refere-se a uma dieta onde prevalecem alimentos de origem vegetal. Existe uma gama alargada de formas de vegetarianismo em que pode haver inclusão de produtos de origem animal, como ovos ou laticínios. Os alimentos comuns às diversas dietas vegetarianas são os cereais, hortícolas, fruta, leguminosas, frutos de casca rija e sementes (14). A inclusão ou exclusão de determinado alimento de origem animal ou a sua exclusão na totalidade é o que confere o fator diferenciador das diversas dietas vegetarianas, tais como $(1,14-16)$ :

- Ovolactovegetariana - elimina o consumo de carne e pescado, mas permite ovos e laticínios;

- Lactovegetariana - exclui carne, pescado e ovos, permitindo laticínios;

- Ovovegetariana - exclui carne, pescado, laticínios, permite ovos;

- Vegetariana estrita e vegana - exclui todos os alimentos de origem animal.

Embora não exista uma definição consensual de semivegetarianismo, existem formas complementares que admitem o consumo ocasional de outros produtos (16-18):

- Pescetariana - exclui o consumo de carne, ovos e laticínios, mas permite o consumo de peixe;

- Flexitariana-Inclui ocasionalmente carne e peixe sendo que o consumo de carne e peixe é mais reduzido comparativamente às dietas com predominância de proteína animal.

A comparação de diversos modelos alimentares em termos de contribuição para uma alimentação saudável, revela maior contributo no caso de dietas omnívoras mas com menor presença de alimentos de origem animal $(19,20)$. Tal padrão de resultados é também encontrado no caso da correspondência entre os mesmos modelos de consumo e a utilização de alimentos incluídos na Dieta Mediterrânica (16, 21). Este aspeto pode ser especialmente interessante para efeito de políticas orientadoras de uma alimentação saudável, ao mesmo tempo que reforça o valor da Dieta Mediterrânica, tentando a síntese entre hábitos culturais e práticas alimentares, e a transição para sistemas de produção alimentar ambientalmente mais sustentáveis.

\section{Alimentação de Base Vegetal e os seus Benefícios para a Saúde Humana}

Num trabalho publicado em 2020 (22) foram analisadas onze diretrizes de alimentação saudável para a população vegetariana em todo o mundo. Cinco grupos de alimentos estão sempre presentes: hortícolas, frutas, cereais e produtos derivados, leguminosas e produtos de soja e frutos de casca rija. Face ao consumo reduzido de produtos de origem animal, a suplementação de vitamina B12 consta em cinco diretrizes.

A condição de dieta baseada em produtos de origem vegetal não é, só por si, suficiente para se assumir um valor protetor da saúde, pois a presença de alimentos vegetais refinados ou ricos em hidratos de carbono de absorção rápida, e de gorduras hidrogenadas, pode ter um efeito oposto sobre a saúde (23-25). O consumo sem controlo do valor energético pode não produzir o efeito de controlo ponderal enunciado por vários trabalhos (26). Devem ser considerados aspetos de composição nutricional e respeitadas as necessidades energéticas e nutricionais de diferentes grupos da população.

Diversos estudos apontam para a existência de dados objetivos enunciados na Tabela 1, como a associação a menor incidência de doença crónica, por comparação com outras dietas, como as dietas onde a presença alimentos de origem animal é significativa (27-29), mas também a perceção que cada indivíduo tem dos benefícios dessas escolhas alimentares, e que determina o comportamento de escolha e consumo alimentar (30). Na Tabela 1 sistematizam-se diversos trabalhos que estudaram os potenciais benefícios (objetivos ou percecionados) que uma DBP pode oferecer. Outros benefícios objetivos verificados são a redução da massa gorda, menor probabilidade de desenvolver cancro, diminuição do estado depressivo, melhoria da saúde cardiovascular, melhoria do humor e melhoria da qualidade de vida. Os benefícios apenas percecionados, são o controlo de disfunções sociais e a diminuição da ansiedade. 
Tabela 1

Potenciais benefícios objetivos ou percecionados de uma DBP para a saúde, bem-estar e satisfação (Adaptado de Fehér et al., 2020) (30)

\begin{tabular}{|c|c|c|c|}
\hline POTENCIAIS BENEFÍCIOS & $\begin{array}{l}\text { BENEFICIO OBJETIVO OU } \\
\text { PERCECIONADO }\end{array}$ & CONCLUSÕES & REFERÊNCIAS \\
\hline \multicolumn{4}{|c|}{ Benefícios para a saúde humana } \\
\hline $\begin{array}{l}\text { Presença significativa de } \\
\text { nutrientes essenciais }\end{array}$ & Objetivo & $\begin{array}{l}\text { A presença de magnésio, de potássio, de ácido fólico, de fibra, de antioxidantes e de } \\
\text { fitoquímicos é superior em DBP comparativamente a dietas com predominância de } \\
\text { proteína animal. }\end{array}$ & $(30-32)$ \\
\hline $\begin{array}{l}\text { Menor ingestão de gorduras } \\
\text { saturadas }\end{array}$ & Percecionado & $\begin{array}{l}\text { As DBP apresentam menor quantidade de gorduras saturadas que as dietas de base } \\
\text { animal. }\end{array}$ & $(33,34)$ \\
\hline Redução da massa gorda & Objetivo & $\begin{array}{l}\text { Por comparação, homens e mulheres vegetarianos apresentam menor massa gorda que } \\
\text { os pares não vegetarianos. }\end{array}$ & $(24,35,36)$ \\
\hline \multirow{3}{*}{$\begin{array}{l}\text { Redução da incidência } \\
\text { de doenças crónicas }\end{array}$} & Objetivo & $\begin{array}{l}\text { As dietas vegetariana e vegana associam-se a menor incidência de diabetes do tipo } 2 \text {, } \\
\text { hipertensão, obesidade e doenças cardíacas. }\end{array}$ & $(37,38)$ \\
\hline & Objetivo & $\begin{array}{l}\text { O consumo de cerca de } 800 \mathrm{~g} / \mathrm{d} \text { de hortofrutícolas, } 225 \mathrm{~g} / \mathrm{d} \text { de grãos integrais e } 15-20 \\
\mathrm{~g} / \mathrm{d} \text { de frutos de casca rija foram consideradas quantidades ótimas para a prevenção } \\
\text { de doenças crónicas. }\end{array}$ & (39) \\
\hline & Objetivo & $\begin{array}{l}\text { Maior adesão aos padrões alimentares baseados em plantas foi associada a um menor } \\
\text { risco de diabetes tipo } 2 \text {. }\end{array}$ & (40) \\
\hline $\begin{array}{l}\text { Menor probabilidade de } \\
\text { desenvolver cancro }\end{array}$ & Objetivo & As DBP podem reduzir o risco de cancro da mama e de cancro da próstata. & $(41,42)$ \\
\hline Melhoria da saúde cardiovascular & Objetivo & $\begin{array}{l}\text { As DBP têm sido associadas com um menor risco de doenças cardíacas e com uma } \\
\text { melhoria no perfil de risco. }\end{array}$ & $(23,25,43,44)$ \\
\hline \multicolumn{4}{|c|}{ Benefícios relacionados com o bem-estar e satisfação } \\
\hline $\begin{array}{l}\text { Pode ajudar no controlo de } \\
\text { disfunções sociais }\end{array}$ & Percecionado & $\begin{array}{l}\text { Através de avaliação por questionário, os consumidores de DBP revelam maior controlo } \\
\text { de disfunções sociais. }\end{array}$ & (45) \\
\hline Melhoria da qualidade de vida & Objetivo & $\begin{array}{l}\text { A adoção de DBP associa-se a redução das despesas em saúde e ao mesmo tempo a } \\
\text { qualidade de vida melhora. }\end{array}$ & $(46-48)$ \\
\hline Melhoria do estado do humor & Objetivo & $\begin{array}{l}\text { A presença de carotenóides e polifenóis em alimentos de origem vegetal, parece estar } \\
\text { relacionada com o aumento da serotonina e com a diminuição dos biomarcadores } \\
\text { inflamatórios (IL-6 e TNF-a), assim como a presença de ácido ascórbico (vitamina C), } \\
\text { muito comum em frutas frescas e vegetais, que interage com os sistemas serotonérgico, } \\
\text { dopaminérgico e noradrenérgico. }\end{array}$ & (49) \\
\hline Diminuição do estado depressivo & Objetivo & $\begin{array}{l}\text { A ingestão de produtos hortofrutícolas está inversamente relacionada com o estado } \\
\text { depressivo. }\end{array}$ & $(50,51)$ \\
\hline $\begin{array}{l}\text { Diminuição de ansiedade e } \\
\text { melhoria do humor }\end{array}$ & Percecionado & Os indivíduos veganos reportaram menos stress e ansiedade do que os omnívoros. & $(52-55)$ \\
\hline
\end{tabular}

DBP: Dieta Baseada em Plantas

\section{Alimentação Baseada em Plantas e Sustentabilidade}

A Estratégia do Prado ao Prato da União Europeia (2020), engloba uma abordagem abrangente da sustentabilidade alimentar, num quadro global de promoção de estilos de vida, saúde e ambiente. É uma perspetiva holística dirigida para a construção de uma cadeia alimentar que gere benefícios para os produtores, os consumidores, o clima e o ambiente. Esta estratégia traduz-se em várias linhas de ação, nomeadamente assegurar uma produção alimentar sustentável (promotora da redução de emissão de gases com efeito de estufa associada à agricultura e pecuária), promover o consumo e facilitar a transição para regimes alimentares saudáveis e sustentáveis e reduzir as perdas e o desperdício alimentar. As DBP parecem alinhar-se com este quadro global de iniciativas políticas e sociais (56).

Ao mesmo tempo que surgem evidências e iniciativas de valorização das dietas baseadas em plantas para a saúde humana, são debatidas preocupações com a qualidade dos sistemas de produção alimentar, de modo a salvaguardar que o aumento do consumo daqueles produtos alimentares e a inerente pressão sobre os sistemas produtivos seja acompanhada de métodos de produção seguros em termos de saúde humana e ambiental (57).

A crescente preocupação sobre o modo de produção por parte dos diversos atores da sociedade, tem vindo a contribuir para o aumento da área de produção e dos investimentos realizados em Portugal, no que diz respeito à área destinada à agricultura biológica (58). Recentemente, foi apresentada uma proposta de modelo pictórico representando a interligação entre a agricultura biológica, a sustentabilidade (social, económica e ambiental) e a saúde pública, questionando a possibilidade de, no futuro, a agricultura biológica poder vir a ser considerada um potencial indicador de saúde pública (59).

\section{Os Produtos Alternativos a Alimentos de Origem Animal: as Soluções da Indústria e as Exigências do Consumidor}

Na sociedade de consumo em que vivemos, a procura modela a oferta e nesta fase o sistema agro-industrial limita-se a responder a essa procura, tendencialmente de mais alimentos de origem vegetal. Uma variedade de alternativas à carne e ao leite estão disponíveis e são amplamente aceites e usadas em dietas vegetarianas e veganas, enquanto outros produtos como substitutos de queijo, ovo e de peixe estão em desenvolvimento. Em 2021 foi publicado um artigo de revisão que apresenta a descrição, as vantagens e as desvantagens de todas essas alternativas (60). Além disso, neste trabalho são apresentadas tendências do consumo e do mercado relativamente às DBP, bem como, os desafios para os consumidores, sustentabilidade, nutrição e saúde.

No mercado já existem as soluções tradicionais para os consumidores vegetarianos, como o tofu, o seitan ou o tempeh. A pesquisa e o desenvolvimento continuam a decorrer com o objetivo de encontrar novas alternativas, existindo já disponíveis análogos de hambúrgueres, salsichas, bifes, nuggets, entre outros. A oferta de produtos análogos da carne, as alternativas aos lacticínios e os alimentos e bebidas enriquecidos com proteínas de origem vegetal está a crescer exponencialmente no mercado atual $(61,62)$.

Contudo, na composição daqueles produtos prevalecem ingredientes críticos, nomeadamente óleos e aditivos alimentares (corantes, conservantes, intensificadores de sabor) o que tem suscitado dúvidas, acelerando assim a procura por soluções menos refinadas $(63,64)$ e 
de clean label (produtos 100\% naturais, livres de transgénicos e sem aditivos) (65). Um típico produto processado substituto de carne, como os anteriormente referidos, contém na sua composição, para além da proteína texturizada e não texturizada, uma quantidade relevante de água, gorduras, intensificadores de sabor, corantes, agentes de ligação e sal (66).

Os mais recentes esforços desenvolvidos neste âmbito são para encontrar substâncias/ingredientes que permitam conferir similaridades nos produtos que pretendam recriar os produtos cárneos, em características específicas, como textura, sabor, aparência e aspetos nutricionais, sendo um requisito adicional para parte dos consumidores que os alimentos sejam menos refinados para ir ao encontro das expectativas do consumidor (66).

As proteínas vegetais mais usadas nos produtos substitutos de carne no mercado são o glúten, de soja e de ervilha. Estes ingredientes têm em comum a elevada disponibilidade e um custo moderado, o que leva a sua maior utilização, além de apresentarem funcionalidades tecnológicas adequadas. Para além das referidas fontes de proteína de origem vegetal, a utilização de microalgas e fungos como fonte de proteína apresentam-se como alternativas viáveis (67).

Paralelamente, será de salientar o potencial de Portugal para a produção de algas, apresentando uma elevada diversidade destas devido à sua localização geográfica, estando muitas espécies já disponíveis no mercado nacional para consumo humano (68), devido ao interesse e potencial utilização das diferentes algas enquanto alimento, suplemento alimentar ou nutracêutico $(69,70)$.

Em Portugal, diversas empresas multinacionais e outras nacionais oferecem já uma panóplia de produtos análogos de carne e de laticínios (71). No entanto, é de realçar que a oferta destes produtos não está livre de batalhas judiciais onde os produtores dos primeiros procuram retardar o uso de qualquer léxico que leve o consumidor a associar produtos de origem vegetal como análogos, como comprova a decisão judicial do Tribunal de Justiça da União Europeia que limita o uso dos termos leite, manteiga, queijo, iogurte e nata só a produtos de origem animal (72). Por outro lado, o tribunal europeu aceitou a permissão de termos como veggie burger e veggie sausage (73).

\section{ANÁLISE CRÍTICA}

As DBP têm vindo rapidamente a ganhar popularidade à medida que os potenciais ou efetivos benefícios da sua adoção na saúde, no bem-estar e no humor do indivíduo, têm vindo a ser demonstrados. As novas gerações são influenciadas pelos meios de comunicação e redes sociais, seguindo formadores de opinião não necessariamente próximos de si, mas espalhados pelo planeta, como meio disseminador. As forças que influenciam a mudança alimentar individual incluem argumentos morais, de identidade cultural, de saúde, de sustentabilidade ambiental, e institucionais (incluindo fatores de ordem económica, social e política) (74). Alguns trabalhos realizados em Portugal evidenciam o peso cultural da presença de carne na alimentação $(75,76)$ e as conceções assentes na falta de informação sobre o valor nutricional de alimentos como as leguminosas (77). Um desafio que se coloca a propósito das DBP, nomeadamente no caso dos consumidores portugueses, é o aprofundamento do conhecimento sobre os argumentos invocados para a adoção de uma alimentação mais centrada em alimentos de origem vegetal, bem como o conhecimento sobre as eventuais barreiras à mudança.

Outro importante desafio é a informação aos consumidores. É fundamental que os argumentos em torno das DBP sejam apresentados de forma clara e esclarecedora (78) para que cada consumidor faça uma escolha alimentar consciente, sem preconceitos e realista quanto ao contributo real para a sua saúde. A rotulagem dos novos produtos alimentares é essencial para o esclarecimento do consumidor que quer adotar substitutos de alimentos animais, pelo que as batalhas judidiciais podem (des)acelerar a conversão destes consumidores. Esta consciência já levou a que uma sentença num tribunal norte-americano favorável ao uso do termo manteiga em produtos vegetais (79).

Os incentivos criados por instituições internacionais envolvidas na definição e implementação de políticas de promoção de saúde, estimula a legitimação deste padrão alimentar que, acredita-se, possibilitará uma melhoria ao nível da saúde pública, através da reformulação dos hábitos de consumo atuais, suportada pelas evidências do efeito protetor que as DBP conferem. Reconhece-se, no entanto, que são necessários mais estudos alargados para melhor compreensão dos efeitos de uma DBP e a relação com outros hábitos de vida promotores de saúde física e mental, incluindo a atividade física. A expansão da adoção destas DBP a mais consumidores e num período alargado, poderá permitir validar os resultados em termos de benefícios para a saúde e os efeitos no sistema agro-alimentar.

A simples adoção de uma DBP não a torna inerentemente mais favorável à saúde ou mais sustentável em termos ambientais, dependendo da sua composição (23-25), e das práticas de produção e os circuitos de comercialização $(78,80)$. O potencial contributo das DBP para a melhoria da saúde das populações, para a sustentabilidade económica e ambiental e, em particular, para a sustentabilidade do sistema alimentar, é reconhecido (81), e inspira novos desafios em termos de tecnologias de produção e economia circular (82), ou mesmo de recurso a alimentos que funcionem como substitutos de fontes proteicas tradicionais como a carne $(82,83)$.

Com a adoção alargada e continuada destas dietas, a influência sobre o sistema agro-alimentar, a começar pela maior procura de matéria-prima de origem vegetal, levará a profundas alterações da produção agrícola, com resposta ambiental que parece ser favorável, dada a redução da exigência de terra arável associada à redução do consumo de carne e substituição pelo consumo de vegetais (84). Contudo, essa adoção será condicionada à oferta no mercado, sempre dependente da dinâmica da economia num mundo cada vez mais globalizado. A indústria procura responder a uma tentativa de balanço entre um consumo mais baseado em produtos vegetais e em produtos com menor pegada ecológica, e hábitos de consumo culturalmente assentes em produtos de origem animal.

\section{CONCLUSÕES}

A revisão da literatura realizada no âmbito deste trabalho sugere que a alimentação de base vegetal está associada a benefícios para a saúde humana, nomeadamente na prevenção de doenças crónicas e no potencial contributo para o bem-estar. Contudo, este argumento deve ser acompanhado da ponderação sobre os alimentos usados e a sua composição nutricional, e sobre as necessidades energéticas e nutricionais dos consumidores.

A adoção de DBP gera um contributo favorável à sustentabilidade ambiental e dos sistemas de produção alimentar, nomeadamente a redução de emissão de gases com efeito de estufa associada à agricultura e pecuária, e a redução das perdas e do desperdício alimentar.

A alteração do padrão de consumo alimentar, com incremento de alimentos de origem vegetal, levanta um conjunto de desafios nas áreas da saúde, do consumo, da informação ao consumidor, da produção agro-alimentar e do desenvolvimento de novos produtos clean label. Em particular, ressalta a necessidade de inovação em produtos alternativos aos produtos de origem animal, tais como, alimentos de base proteica vegetal, produtos análogos da carne, alternativas aos laticínios, e alimentos e bebidas enriquecidos com proteínas de origem vegetal. 


\section{AGRADECIMENTOS}

Financiamento pelo projecto EQVEGAN - European Qualifications \& Competences for the Vegan Food Industry 621581-EPP-1-2020-1-PTEPPKA2-SSA-EQVEGAN.

\section{REFERÊNCIAS BIBLIOGRÁFICAS}

1. Silva SCG, Pinho JP, Borges C, Santos CT, Santos A, Graça P. Linhas de Orientação para uma Alimentação Vegetariana Saudável [Internet]. Programa Nacional para a Promoção da Alimentação Saudável. 2015. 1-50 p. Disponível em: https://docs. google.com/viewer?url=https\%3A\%2F\%2Frepositorio-aberto.up.pt\%2Fbitstream\%2F10216\%2F80821\%2F2\%2F123855.pdf.

2. Zampa M. How Many Vegans Are There Really in the U.S.? [Internet]. 2019. Disponível em: https://sentientmedia.org/how-many-vegans-are-there-in-the-u-s/.

3. Nielsen Portugal. Vegetarianos em Portugal [Internet]. 2017. Disponível em: https:// www.centrovegetariano.org/Article-620-Numero-vegetarianos-quadruplica-10-anos-Portugal.html.

4. Nielsen Portugal. Vegetarianos em Portugal [Internet]. 2007. Disponível em: https:// www.centrovegetariano.org/Article-451-Portugal-quantos-Vegetarianos-30-mil.html. 5. Lantern. The Green Revolution Portugal. Junho de 2020 [citado 20 de Agosto de 2021]; Disponível em: http://www.lantern.es.

6. Assembleia da República. Lei n.o 11/2017 de 17 de abril. Diário da República [Internet]. 2017;1a série(75):1974. Disponível em: https://dre.pt/pesquisa/-/search/106886578/details/normal?q=Lei+n.o 11\%2F2017\%2C de+17+de+abril.

7. EACEA. European Qualification \& Competences for the Vegan Food Industry. 2020 8. Pimentel D, Pimentel M. Sustainability of meat-based and plant-based diets and the environment. Am J Clin Nutr [Internet]. 1 de Setembro de 2003 [citado 19 de Agosto de 2021];78(3):660S-663S. Disponível em: https://academic.oup.com/ajcn/ article/78/3/660S/4690010.

9. Nicholls J, Drewnowski A. Toward Sociocultural Indicators of Sustainable Healthy Diets. Sustain 2021, Vol 13, Page 7226 [Internet]. 28 de Junho de 2021 [citado 19 de Agosto de 2021];13(13):7226. Disponível em: https://www.mdpi.com/20711050/13/13/7226/htm.

10. Ostfeld RJ. Definition of a plant-based diet and overview of this special issue. $J$ Geriatr Cardiol. 2017;14(5):315.

11. Harvard Men's Health Watch. The right plant-based diet for you. Harvard Univ [Internet]. 2018; Disponível em: https://www.health.harvard.edu/staying-healthy/the-right-plant-based-diet-for-you.

12. Williams KA, Patel H. Healthy Plant-Based Diet: What Does it Really Mean? J Am Coll Cardiol. 2017;70(4):423-5.

13. Beverland MB. Sustainable Eating: Mainstreaming Plant-Based Diets In Developed Economies. http://dx.doi.org/101177/0276146714526410 [Internet]. 5 de Março de 2014 [citado 19 de Agosto de 2021];34(3):380. Disponível em: https://journals.sagepub.com/doi/abs/10.1177/0276146714526410.

14. Craig, W Mangels A. Position of the American Dietetic Association: Vegetarian Diets. J Am Diet Assoc [Internet]. Julho de 2009;109(7):1266-82. Disponível em: https:// linkinghub.elsevier.com/retrieve/pii/S0002822309007007.

15. Position of the American Dietetic Association and Dietitians of Canada: Vegetarian Diets. Can J Diet Pract Res [Internet]. Julho de 2003;64(2):62-81. Disponível em: https://dcjournal.ca/doi/10.3148/64.2.2003.62.

16. Clarys P, Deliens T, Huybrechts I, Deriemaeker P, Vanaelst B, De Keyzer W, et al Comparison of Nutritional Quality of the Vegan, Vegetarian, Semi-Vegetarian, Pesco-Vegetarian and Omnivorous Diet. Nutrients [Internet]. 24 de Março de 2014;6(3):1318-32. Disponível em: http://www.mdpi.com/2072-6643/6/3/1318.

17. Groufh-Jacobsen S, Hess SY, Aakre I, Folven Gjengedal EL, Blandhoel Pettersen K, Henjum S. Vegans, Vegetarians and Pescatarians Are at Risk of lodine Deficiency in Norway. Nutrients [Internet]. 20 de Novembro de 2020;12(11):3555. Disponível em: https://www.mdpi.com/2072-6643/12/11/3555.

18. Derbyshire EJ. Flexitarian Diets and Health: A Review of the Evidence-Based Literature. Front Nutr. 6 de Janeiro de 2017;3.

19. Krebs-Smith SM, Pannucci TRE, Subar AF, Kirkpatrick SI, Lerman JL, Tooze JA, et al. Update of the Healthy Eating Index: HEl-2015. J Acad Nutr Diet. 2018;118(9):1591602.

20. Magkos F, Tetens I, Bügel SG, Felby C, Schacht SR, Hill JO, et al. The Environmental Foodprint of Obesity [Internet]. Vol. 28, Obesity. John Wiley \& Sons, Ltd; 2020 [citado 20 de Agosto de 2021]. p. 73-9. Disponível em: https://onlinelibrary.wiley.com/ doi/full/10.1002/oby.22657.

21. Trichopoulou A, Costacou T, Bamia C, Trichopoulos D. Adherence to a Mediterranean Diet and Survival in a Greek Population. N Engl J Med [Internet]. 26 de Junho de 2003;348(26):2599-608. Disponível em: http://www.nejm.org/doi/abs/10.1056/ NEJMoa025039.

22. Gai Costantino C, Morales Morante LF. Vegetarian dietary guidelines: a comparative dietetic and communicational analysis of eleven international pictorial representations. Rev Española Nutr Humana y Dietética [Internet]. 16 de Julho de 2020;24(2):120. Disponível em: http://renhyd.org/index.php/renhyd/article/view/953.

23. Baden MY, Liu G, Satija A, Li Y, Sun Q, Fung TT, et al. Changes in Plant-Based Diet Quality and Total and Cause-Specific Mortality. Circulation [Internet]. 17 de Setembro de 2019 [citado 20 de Agosto de 2021];140(12):979-91. Disponível em: https://pubmed. ncbi.nlm.nih.gov/31401846/.

24. Satija A, Malik V, Rimm EB, Sacks F, Willett W, Hu FB. Changes in intake of plant-based diets and weight change: Results from 3 prospective cohort studies [Internet]. Vol. 110, American Journal of Clinical Nutrition. 2019 [citado 20 de Agosto de 2021]. p. 574-82. Disponível em: https://academic.oup.com/ajcn/.

25. Satija A, Hu FB. Plant-based diets and cardiovascular health [Internet]. Vol. 28, Trends in Cardiovascular Medicine. Trends Cardiovasc Med; 2018 [citado 19 de Agosto de 2021]. p. 437-41. Disponível em: https://pubmed.ncbi.nlm.nih.gov/29496410/.

26. Magkos F, Tetens I, Bügel SG, Felby C, Schacht SR, Hill JO, et al. A Perspective on the Transition to Plant-Based Diets: A Diet Change May Attenuate Climate Change, but Can It Also Attenuate Obesity and Chronic Disease Risk. Vol. 11, Advances in Nutrition. Oxford University Press; 2020. p. 1-9.

27. Ornish D, Brown SE, Billings JH, Scherwitz LW, Armstrong WT, Ports TA, et al. Can lifestyle changes reverse coronary heart disease? Lancet [Internet]. Julho de 1990;336(8708):129-33. Disponível em: https://linkinghub.elsevier.com/retrieve/ pii/014067369091656U.

28. Pinho JP, Silva SCG, Borges C, Santos CT, Santos A, Guerra A, et al. Alimentação Vegetariana Em Idade Escolar. Programa Nac para a Promoção da Aliment Saudável - Direção-Geral da Saúde [Internet]. 2016;60. Disponível em: http://www.apn.org.pt/ documentos/efemerides/Alimentacao-Vegetariana-em-Idade-Escolar-.pdf.

29. Chahoud G, Aude YW, Mehta JL. Dietary recommendations in the prevention and treatment of coronary heart disease: Do we have the ideal diet yet? Am J Cardiol [Internet]. Novembro de 2004;94(10):1260-7. Disponível em: https://linkinghub.elsevier. com/retrieve/pii/S0002914904012238.

30. Fehér A, Gazdecki M, Véha M, Szakály M, Szakály Z. A Comprehensive Review of the Benefits of and the Barriers to the Switch to a Plant-Based Diet. Sustainability [Internet]. 19 de Maio de 2020;12(10):4136. Disponível em: https://www.mdpi. com/2071-1050/12/10/4136.

31. Dwyer JT. Health aspects of vegetarian diets. Am J Clin Nutr [Internet]. 1 de Setembro de 1988;48(3):712-38. Disponível em: https://academic.oup.com/ajcn/ article/48/3/712-738/4716406.

32. Pomerleau J, McKee M, Lobstein T, Knai C. The burden of disease attributable to nutrition in Europe. Public Health Nutr [Internet]. 22 de Agosto de 2003;6(5):453-61. Disponível em: https://www.cambridge.org/core/product/identifier/S1368980003000624/ type/journal_article.

33. Lea E, Worsley A. Benefits and barriers to the consumption of a vegetarian diet in Australia. Public Health Nutr [Internet]. 22 de Agosto de 2003;6(5):505-11. Disponível em: https://www.cambridge.org/core/product/identifier/S1368980003000685/ type/journal_article.

34. Lea EJ, Crawford D, Worsley A. Public views of the benefits and barriers to the consumption of a plant-based diet. Eur J Clin Nutr [Internet]. 1 de Julho de 2006;60(7):828837. Disponível em: http://www.nature.com/articles/1602387.

35. Barnard ND, Cohen J, Jenkins DJ, Turner-McGrievy G, Gloede L, Green A, et 
al. A low-fat vegan diet and a conventional diabetes diet in the treatment of type 2 diabetes: a randomized, controlled, 74-wk clinical trial. Am J Clin Nutr [Internet]. 1 de Maio de 2009;89(5):1588S-1596S. Disponível em: https://academic.oup.com/ajcn/ article/89/5/1588S/4596944.

36. Berkow SE, Barnard N. Vegetarian Diets and Weight Status. Nutr Rev [Internet] Abril de 2006;64(4):175-88. Disponível em: https://academic.oup.com/nutritionreviews/article-lookup/doi/10.1111/j.1753-4887.2006.tb00200.x.

37. Melina V, Craig W, Levin S. Position of the Academy of Nutrition and Dietetics: Vegetarian Diets. J Acad Nutr Diet [Internet]. Dezembro de 2016;116(12):1970-80. Disponível em: https://linkinghub.elsevier.com/retrieve/pii/S2212267216311923.

38. Massera D, Zaman T, Farren GE, Ostfeld RJ. A Whole-Food Plant-Based Die Reversed Angina without Medications or Procedures. Case Reports Cardiol [Internet] 2015;2015:1-3. Disponível em: http://www.hindawi.com/journals/cric/2015/978906/. 39. Aune D. Plant Foods, Antioxidant Biomarkers, and the Risk of Cardiovascular Disease, Cancer, and Mortality: A Review of the Evidence. Adv Nutr [Internet]. 1 de Novembro de 2019 [citado 19 de Agosto de 2021];10(Supplement_4):S404-21. Disponíve em: https://academic.oup.com/advances/article/10/Supplement_4/S404/5624065. 40. Qian F, Liu G, Hu FB, Bhupathiraju SN, Sun Q. Association Between Plant-Based Dietary Patterns and Risk of Type 2 Diabetes: A Systematic Review and Meta-analysis. JAMA Intern Med [Internet]. 1 de Outubro de 2019 [citado 19 de Agosto de 2021];179(10):1335-44. Disponível em: https://jamanetwork.com/journals/jamainternalmedicine/fullarticle/2738784.

41. Nechuta SJ, Caan BJ, Chen WY, Lu W, Chen Z, Kwan ML, et al. Soy food intake after diagnosis of breast cancer and survival: an in-depth analysis of combined evidence from cohort studies of US and Chinese women. Am J Clin Nutr [Internet] 1 de Julho de 2012;96(1):123-32. Disponível em: https://academic.oup.com/ajcn/ article/96/1/123/4571417.

42. Richman EL, Stampfer MJ, Paciorek A, Broering JM, Carroll PR, Chan JM. Intakes of meat, fish, poultry, and eggs and risk of prostate cancer progression. Am J Clin Nutr [Internet]. 1 de Março de 2010;91(3):712-21. Disponível em: https://academic.oup. com/ajcn/article/91/3/712/4597223.

43. Alasmre FA, Alotaibi HA. Plant-Based Diet: A Potential Intervention for Heart Failure. Cureus [Internet]. 25 de Maio de 2020; Disponível em: https://www.cureus.com/ articles/29885-plant-based-diet-a-potential-intervention-for-heart-failure.

44. Najjar RS, Moore CE, Montgomery BD. Consumption of a defined, plant-based diet reduces lipoprotein(a), inflammation, and other atherogenic lipoproteins and particles within 4 weeks. Clin Cardiol [Internet]. Agosto de 2018;41(8):1062-8. Disponível em: https://onlinelibrary.wiley.com/doi/10.1002/clc.23027.

45. Judge M, Wilson MS. Vegetarian Utopias: Visions of dietary patterns in future societies and support for social change. Futures [Internet]. Agosto de 2015;71:57-69. Disponivel em: https://linkinghub.elsevier.com/retrieve/pii/S0016328715000890.

46. Kökény T. The History of Vegetarianism in Hungary. Társadalomkutatás [Internet] Junho de 2009;27(2):203-25. Disponível em: https://akjournals.com/doi/10.1556/ tarskut.27.2009.2.7.

47. Meyer TE, Kovács SJ, Ehsani AA, Klein S, Holloszy JO, Fontana L. Long-Term Caloric Restriction Ameliorates the Decline in Diastolic Function in Humans. J Am Coll Cardiol [Internet]. Janeiro de 2006;47(2):398-402. Disponível em: https://linkinghub. elsevier.com/retrieve/pii/S0735109705025775.

48. Toumpanakis A, Turnbull T, Alba-Barba I. Effectiveness of plant-based diets in promoting well-being in the management of type 2 diabetes: a systematic review. BMJ Open Diabetes Res Care [Internet]. Novembro de 2018;6(1):e000534. Disponível em: https://drc.bmj.com/lookup/doi/10.1136/bmjdrc-2018-000534.

49. Arab A, Mehrabani S, Moradi S, Amani R. The association between diet and mood: A systematic review of current literature. Psychiatry Res [Internet]. Janeiro de 2019;271:428-37. Disponivel em: https://linkinghub.elsevier.com/retrieve/pii/ S0165178118307583.

50. Liu X, Yan Y, Li F, Zhang D. Fruit and vegetable consumption and the risk of depression: A meta-analysis. Nutrition [Internet]. Março de 2016;32(3):296-302. Disponível em: https://linkinghub.elsevier.com/retrieve/pii/S0899900715003974.

51. AlAmmar WA, Albeesh FH, Khattab RY. Food and Mood: the Corresponsive Effect
[Internet]. Vol. 9, Current Nutrition Reports. Springer; 2020 [citado 29 de Abril de 2021]. p. 296-308. Disponivel em: https://pubmed.ncbi.nlm.nih.gov/32623655/.

52. Beezhold B, Radnitz C, Rinne A, DiMatteo J. Vegans report less stress and anxiety than omnivores. Nutr Neurosci [Internet]. 21 de Outubro de 2015;18(7):289-96. Disponivel em: http://www.tandfonline.com/doi/full/10.1179/1476830514Y.0000000164. 53. Beezhold BL, Johnston CS. Restriction of meat, fish, and poultry in omnivores improves mood: A pilot randomized controlled trial. Nutr J [Internet]. 14 de Dezembro de 2012;11(1):9. Disponível em: http://nutritionj.biomedcentral.com/articles/10.1186/1475-2891-11-9.

54. Beezhold BL, Johnston CS, Daigle DR. Vegetarian diets are associated with healthy mood states: a cross-sectional study in Seventh Day Adventist adults. Nutr J [Internet]. 1 de Dezembro de 2010;9(1):26. Disponível em: http://nutritionj.biomedcentral.com/ articles/10.1186/1475-2891-9-26.

55. Olabi A, Levitsky DA, Hunter JB, Spies R, Rovers AP, Abdouni L. Food and mood: A nutritional and mood assessment of a 30-day vegan space diet. Food Qual Prefer [Internet]. Março de 2015;40:110-5. Disponível em: https://linkinghub.elsevier.com/ retrieve/pii/S0950329314001888.

56. European Green Deal. Farm to Fork Strategy.For a fair, healthy and environmentally-friendly food system. 2020 [citado 2 de Setembro de 2020];22. Disponível em: https://ec.europa.eu/food/farm2fork_en.

57. Wyckhuys KAG, Aebi A, Bijleveld van Lexmond MFIJ, Bojaca CR, Bonmatin JM, Furlan $L$, et al. Resolving the twin human and environmental health hazards of a plant-based diet. Environ Int. 1 de Novembro de 2020;144:106081.

58. Ferreira J. O presente e o futuro da agricultura biológica. Dossier Agricultura Biológica. Agrotec. 2020;10-3.

59. Ferreira F, Mendes-Moreira P, Botelho G. Is organic agriculture a potential public health indicator? Evidence from literature. Open Agric [Internet]. 31 de Dezembro de 2020;5(1):914-29. Disponível em: https://www.degruyter.com/document/doi/10.1515/ opag-2020-0088/html.

60. Alcorta A, Porta A, Tárrega A, Alvarez MD, Pilar Vaquero M. Foods for plant-based diets: Challenges and innovations [Internet]. Vol. 10, Foods. 2021. Disponível em: https://doi.org/10.3390/foods10020293.

61. A quarter of Brits use plant-based milk | Mintel.com [Internet]. [citado 27 de Abril de 2021]. Disponivel em: https://www.mintel.com/press-centre/food-and-drink/milking-the-vegan-trend-a-quarter-23-of-brits-use-plant-based-milk.

62. GFI. Good Food Retail Report: Benchmarking the Top U.S Retailers on Plant-Based Sales Strategies [Internet]. 2020 [citado 27 de Abril de 2021]. Disponível em: https:// gfi.org/resource/plant-based-retail-report/.

63. Niva M, Vainio A, Jallinoja P. Barriers to Increasing Plant Protein Consumption in Western Populations. Em: Vegetarian and Plant-Based Diets in Health and Disease Prevention [Internet]. Elsevier; 2017. p. 157-71. Disponível em: https://linkinghub. elsevier.com/retrieve/pii/B9780128039687000101.

64. Szejda K, Urbanovich T, Wilks M. Accelerating Consumer Adoption of Plant-Based Meat: An Evidence-Based Guide for Effective Practice. 2020;1-111. Disponível em: go.gfi.org/plant-based-meat-consumer-adoption\%0D.

65. Asioli D, Aschemann-Witzel J, Caputo V, Vecchio R, Annunziata A, Næs T, et al. Making sense of the "clean label" trends: A review of consumer food choice behavior and discussion of industry implications [Internet]. Vol. 99, Food Research International. Elsevier Ltd; 2017 [citado 25 de Maio de 2021]. p. 58-71. Disponível em: https:// pubmed.ncbi.nlm.nih.gov/28784520/.

66. Kyriakopoulou K, Keppler JK, van der Goot AJ. Functionality of Ingredients and Additives in Plant-Based Meat Analogues. Foods [Internet]. 12 de Março de 2021;10(3):29. Disponível em: https://www.mdpi.com/2304-8158/10/3/600.

67. McClements DJ, Weiss J, Kinchla AJ, Nolden AA, Grossmann L. Methods for Testing the Quality Attributes of Plant-Based Foods: Meat- and Processed-Meat Analogs. Foods. 2021;10(2):260.

68. Gaspar R, Pereira L, Sousa-Pinto I. The seaweed resources of Portugal. Bot Mar [Internet]. 1 de Outubro de 2019 [citado 19 de Agosto de 2021];62(5):499-525. Disponível em: https://www.degruyter.com/document/doi/10.1515/bot-2019-0012/html. 69. Paiva L, Lima E, Ferreira Patarra R, Neto Al, Baptista J. Edible Azorean macroalgae 
as source of rich nutrients with impact on human health. 2014 [citado 19 de Agosto de 2021]; Disponível em: http://dx.doi.org/10.1016/j.foodchem.2014.04.119.

70. Araújo R, Vázquez Calderón F, Sánchez López J, Azevedo IC, Bruhn A, Fluch S, et al. Current Status of the Algae Production Industry in Europe: An Emerging Sector of the Blue Bioeconomy. Front Mar Sci. 27 de Janeiro de 2021;0:1247.

71. Pacheco F. Crescimento de consumo de plant-based food? Consumidores e empresas respondem à chamada - Hipersuper - Hipersuper [Internet]. 2021 [citado 20 de Agosto de 2021]. Disponível em: https://www.hipersuper.pt/2021/05/06/crescimento-consumo-plant-based-food-consumidores-empresas-respondem-chamada/. 72. Cornall J. Plant-based products can't use dairy names: European Court of Justice [Internet]. 2017 [citado 20 de Agosto de 2021]. Disponível em: https://www.dairyreporter.com/Article/2017/06/14/Plant-based-products-can-t-use-dairy-names-European-Court-of-Justice.

73. The Guardian. European farmers lose attempt to ban terms such as veggie burger. 2020; Disponível em: https://www.theguardian.com/world/2020/oct/23/european-farmers-lose-attempt-to-ban-terms-such-veggie-burger.

74. Beverland MB. Sustainable Eating. J Macromarketing [Internet]. 5 de Setembro de 2014;34(3):369-82. Disponível em: http://journals.sagepub.com/ doi/10.1177/0276146714526410.

75. Graça J, Calheiros MM, Oliveira A. Attached to meat? (Un)Willingness and intentions to adopt a more plant-based diet. Appetite. 1 de Dezembro de 2015;95:113-25. 76. Graça J, Oliveira A, Calheiros MM. Meat, beyond the plate. Data-driven hypotheses for understanding consumer willingness to adopt a more plant-based diet. Appetite. 1 de Julho de 2015;90:80-90.

77. Duarte M, Vasconcelos M, Pinto E. Pulse Consumption among Portuguese Adults: Potential Drivers and Barriers towards a Sustainable Diet. Nutr 2020, Vol 12, Page 3336 [Internet]. 30 de Outubro de 2020 [citado 20 de Agosto de 2021];12(11):3336. Disponível em: https://www.mdpi.com/2072-6643/12/11/3336/htm.

78. Lonnie M, Johnstone AM. The public health rationale for promoting plant protein as an important part of a sustainable and healthy diet. Nutr Bull [Internet]. 3 de Setembro de 2020;45(3):281-93. Disponível em: https://onlinelibrary.wiley.com/doi/10.1111/ nbu. 12453.

79. Mridul A. Miyoko's Creamery wins plant-based labelling legal battle for its vegan butter. vegan Rev [lnternet]. 2021; Disponível em: https://theveganreview.com/miyokos-creamery-wins-plant-based-labelling-legal-battle-vegan-butter-california-state/.

80. Macdiarmid JI. Is a healthy diet an environmentally sustainable diet? Proc Nutr Soc [Internet]. 28 de Fevereiro de 2013;72(1):13-20. Disponível em: https://www.cambridge.org/core/product/identifier/S0029665112002893/type/journal_article.

81. Sabaté J, Soret S. Sustainability of plant-based diets: back to the future. Am J Clin Nutr [Internet]. 1 de Julho de 2014 [citado 19 de Agosto de 2021];100(suppl_1):476S-482S. Disponível em: https://academic.oup.com/ajcn/article/100/suppl_1/476S/4576675.

82. Sadhukhan J, Dugmore TIJ, Matharu A, Martinez-Hernandez E, Aburto J, Rahman PKSM, et al. Perspectives on "Game Changer" Global Challenges for Sustainable 21st Century: Plant-Based Diet, Unavoidable Food Waste Biorefining, and Circular Economy. Sustainability [Internet]. 5 de Março de 2020;12(5):1976. Disponível em: https://www. mdpi.com/2071-1050/12/5/1976.

83. Hu FB, Otis BO, McCarthy G. Can Plant-Based Meat Alternatives Be Part of a Healthy and Sustainable Diet? JAMA [Internet]. 22 de Outubro de 2019;322(16):1547. Disponivel em: https://jamanetwork.com/journals/jama/fullarticle/2749260.

84. Poore J, Nemecek T. Reducing food's environmental impacts through producers and consumers. Science (80- ). 2018;360(6392):987-92. 